\title{
The UK National Quantum Technologies Hub in sensors and metrology (Keynote Paper)
}

\section{K. Bongs, V. Boyer, M. A. Cruise, A. Freise, M. Holynski, et al.}

K. Bongs, V. Boyer, M. A. Cruise, A. Freise, M. Holynski, J. Hughes, A. Kaushik, Y.-H. Lien, A. Niggebaum, M. Perea-Ortiz, P. Petrov, S. Plant, Y. Singh, A. Stabrawa, D. J. Paul, M. Sorel, D. R. S. Cumming, J. H. Marsh, R. W. Bowtell, M. G. Bason, R. P. Beardsley, R. P. Campion, M. J. Brookes, T. Fernholz, T. M. Fromhold, L. Hackermuller, P. Krüger, X. Li, J. O. Maclean, C. J. Mellor, S. V. Novikov, F. Orucevic, A. W. Rushforth, N. Welch, T. M. Benson, R. D. Wildman, T. Freegarde, M. Himsworth, J. Ruostekoski, P. Smith, A. Tropper, P. F. Griffin, A. S. Arnold, E. Riis, J. E. Hastie, D. Paboeuf, D. C. Parrotta, B. M. Garraway, A. Pasquazi, M. Peccianti, W. Hensinger, E. Potter, A. H. Nizamani, H. Bostock, A. Rodriguez Blanco, G. Sinuco-Leon, I. R. Hill, R. A. Williams, P. Gill, N. Hempler, G. P. A. Malcolm, T. Cross, B. O. Kock, S. Maddox, P. John, "The UK National Quantum Technologies Hub in sensors and metrology (Keynote Paper)," Proc. SPIE 9900, Quantum Optics, 990009 (9 June 2016); doi: 10.1117/12.2232143 


\title{
The UK National Quantum Technology Hub in Sensors and Metrology
}

K. Bongs ${ }^{* a}$, V. Boyer ${ }^{a}$, M.A. Cruise ${ }^{a}$, A. Freise ${ }^{a}$, M. Holynski ${ }^{a}$, J. Hughes ${ }^{a}$, A. Kaushik ${ }^{\mathrm{a}}$, Y.-H.

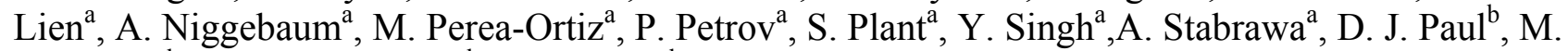
Sorel $^{\mathrm{b}}$, D.R.S. Cumming ${ }^{\mathrm{b}}$, J.H. Marsh ${ }^{\mathrm{b}}$, R.W. Bowtell ${ }^{\mathrm{c}}$, M.G. Bason ${ }^{\mathrm{c}}$, R.P. Beardsley ${ }^{\mathrm{c}}$, R.P. Campion $^{\mathrm{c}}$, M.J. Brookes ${ }^{\mathrm{c}}$, T. Fernholz ${ }^{\mathrm{c}}$, T.M. Fromhold ${ }^{\mathrm{c}}$, L. Hackermuller ${ }^{\mathrm{c}}$, P. Krüger ${ }^{\mathrm{c}}, \mathrm{X}^{\mathrm{X}}$. Li ${ }^{\mathrm{c}}$, J.O.

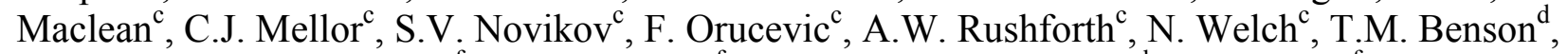
R.D. Wildman ${ }^{\mathrm{e}}$, T. Freegarde ${ }^{\mathrm{f}}$, M. Himsworth ${ }^{\mathrm{f}}$, J. Ruostekoski $^{\mathrm{g}}$, P. Smith ${ }^{\mathrm{h}}$, A. Tropper ${ }^{\mathrm{f}}$, P. F. Griffin ${ }^{\mathrm{i}}$, A. S. Arnold ${ }^{\mathrm{i}}$, E. Riis ${ }^{\mathrm{i}}$, J. E. Hastie $^{\mathrm{j}}$, D. Pabœuf ${ }^{\mathrm{j}}$ D. C. Parrotta ${ }^{\mathrm{j}}$, B.M.Garraway ${ }^{\mathrm{k}}$, A.Pasquazi ${ }^{\mathrm{k}}$, M. Peccianti $^{\mathrm{k}}$, W.Hensinger ${ }^{\mathrm{k}}$, E. Potter ${ }^{\mathrm{k}}$, A.H. Nizamani ${ }^{\mathrm{k}}$, H. Bostock $^{\mathrm{k}}$, A. Rodriguez Blanco ${ }^{\mathrm{k}}$, G. Sinuco-León $^{\text {k, I. R. Hill }}{ }^{1}$, R.A. Williams, ${ }^{1}$ P. Gill ${ }^{1}$, N. Hempler ${ }^{\mathrm{m}}$, G. P. A. Malcolm ${ }^{\mathrm{m}}$, T. Cross ${ }^{\mathrm{n}}$, B. O. $\operatorname{Kock}^{n}$, S. Maddox ${ }^{n}$, P. John ${ }^{\text {n }}$

${ }^{a}$ Midlands Ultracold Atom Research Centre, Physics and Astronomy, University of Birmingham, Birmingham B15 2TT, UK; ' School of Engineering, University of Glasgow, Rankine Building, Oakfield Avenue, Glasgow, G12 8LT, UK; ${ }^{\mathrm{C} S c h o o l ~ o f ~ P h y s i c s ~ a n d ~ A s t r o n o m y, ~ U n i v e r s i t y ~ o f ~}$ Nottingham, Nottingham NG7 2RD, UK; ${ }^{\mathrm{d}}$ George Green Institute for Electromagnetics Research, University of Nottingham, University Park, Nottingham, UK; ${ }^{\mathrm{e}}$ Additive Manufacturing and 3D Printing Research Group, Faculty of Engineering, University of Nottingham, Nottingham NG7 2RD,

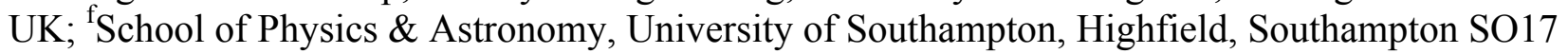
1BJ, UK; ${ }^{\mathrm{g} S c h o o l}$ of Mathematical Sciences, University of Southampton, Highfield, Southampton SO17 1BJ, UK; ' Optoelectronics Research Centre, University of Southampton, Highfield, Southampton SO17 1BJ, UK; ${ }^{\mathrm{i}}$ Physics, University of Strathclyde, Glasgow G4 0NG, UK; ${ }^{\mathrm{j}}$ Institute

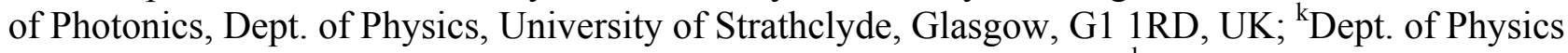
and Astronomy, University of Sussex, Falmer Brighton, BN1 9QJ, UK; ${ }^{1}$ National Physical Laboratory, Teddington, TW11 0LW, UK; ${ }^{m}$ M Squared Lasers Ltd, 1 Kelvin Campus, West of Scotland Science Park, Maryhill Road, Glasgow, G20 0SP, UK; ${ }^{\mathrm{e}} 2 \mathrm{v}, 106$ Waterhouse Lane, Chelmsford, Essex, England, CM1 2QU, UK.

\begin{abstract}
The UK National Quantum Technology Hub in Sensors and Metrology is one of four flagship initiatives in the UK National of Quantum Technology Program. As part of a 20-year vision it translates laboratory demonstrations to deployable practical devices, with game-changing miniaturized components and prototypes that transform the state-ofthe-art for quantum sensors and metrology. It brings together experts from the Universities of Birmingham, Glasgow, Nottingham, Southampton, Strathclyde and Sussex, NPL and currently links to over 15 leading international academic institutions and over 70 companies to build the supply chains and routes to market needed to bring 10-1000x improvements in sensing applications. It seeks, and is open to, additional partners for new application development and creates a point of easy open access to the facilities and supply chains that it stimulates or nurtures.
\end{abstract}

Keywords: Quantum technology, atom interferometry, atomic clocks, gravity sensors, magnetic sensors, quantum optics

Quantum Optics, edited by Jürgen Stuhler, Andrew J. Shields, Proc. of SPIE Vol. 9900 $990009 \cdot$ ? 2016 SPIE · CCC code: 0277-786X/16/\$18 - doi: 10.1117/12.2232143 


\section{INTRODUCTION}

The Hub programme within a 20 year vision (Fig. 1) is about translating lab demonstrations to deployable practical devices with small reliable game changing components and to drive the application space in the area of quantum sensors and metrology.

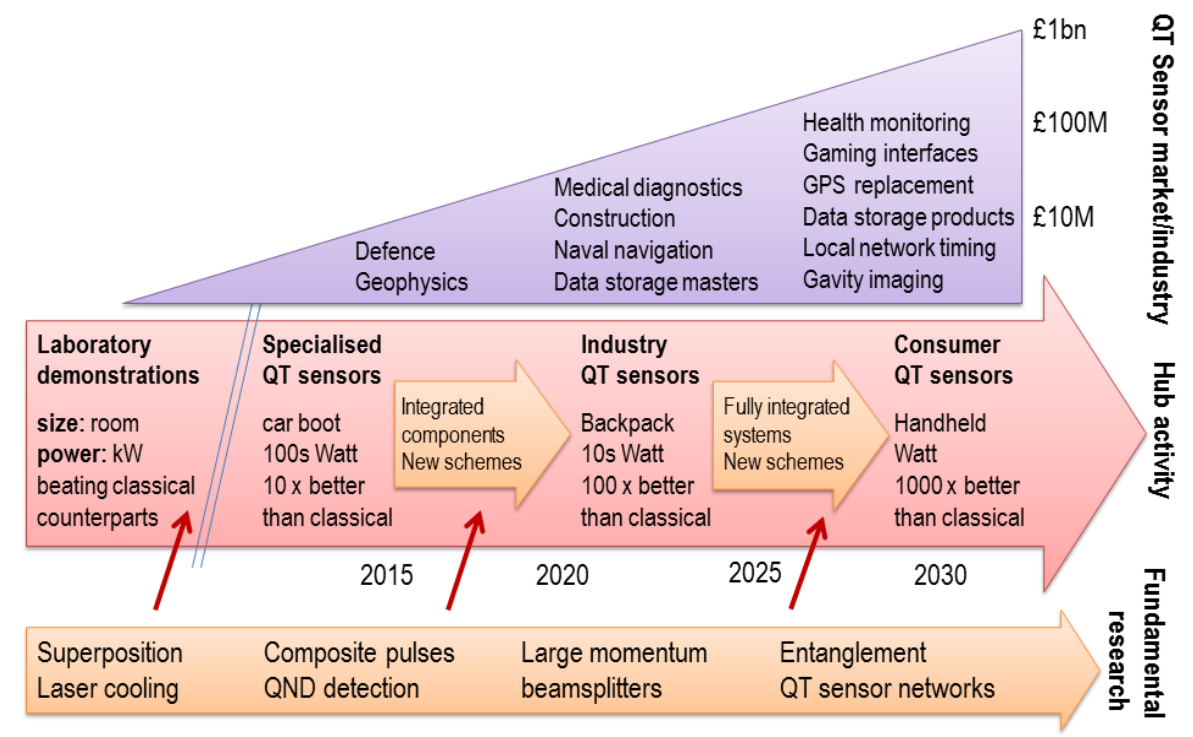

Figure 1. Roadmap for developing Quantum Sensors in a programme linking underpinning fundamental research and industry.

The quantum superposition principle lies at the heart of quantum theory and allows two classically distinct and exclusive alternatives to coexist. The mission of this Quantum technology Hub is to take this well-tested corner stone of quantum mechanics and to provide a five year route to marketable devices that, by exploiting this principle, out-perform conventional sensors.

In general terms all quantum sensors in this Hub are based on the ability of quantum objects to be at two places and/or in two different internal states at the same time. First laser cooling and trapping methods are used to prepare the atoms in a well-controlled motional state. Then tailored laser or microwave pulses are used to create superposition states and recombine them after some "measurement time", leading to interference in final state populations. The arrangement of pulses and laser geometry determines the measurement type, e.g. separating paths vertically: gravity, enclosing an area between the paths: rotation, superposing different spin states: magnetic fields and superposing different energy states: time. The result is read out by state-selective detection of the relative population in the two states at the end.

We have identified five areas where laboratory experiments have shown outperformance of conventional sensors: gravity, rotation, clocks, magnetism and imaging. Our strategy is to develop practical prototypes and demonstrators for each of these technologies. By working in tandem with component manufacturers and systems engineers these demonstrators will be built on a common set of interconnecting quantum components. Communication between businesses along the supply chain is enabled by co-locating university scientists with commercial companies.

In parallel, and equally critical, the Hub is seeding the market with early adopters of these sensor technologies. These projects deliver early examples of the technology for a much wider market to understand the potential.

In the following, we will discuss the individual activity areas within the Hub, creating supply chain technologies, training skilled personnel and building markets as illustrated in fig. 2. 


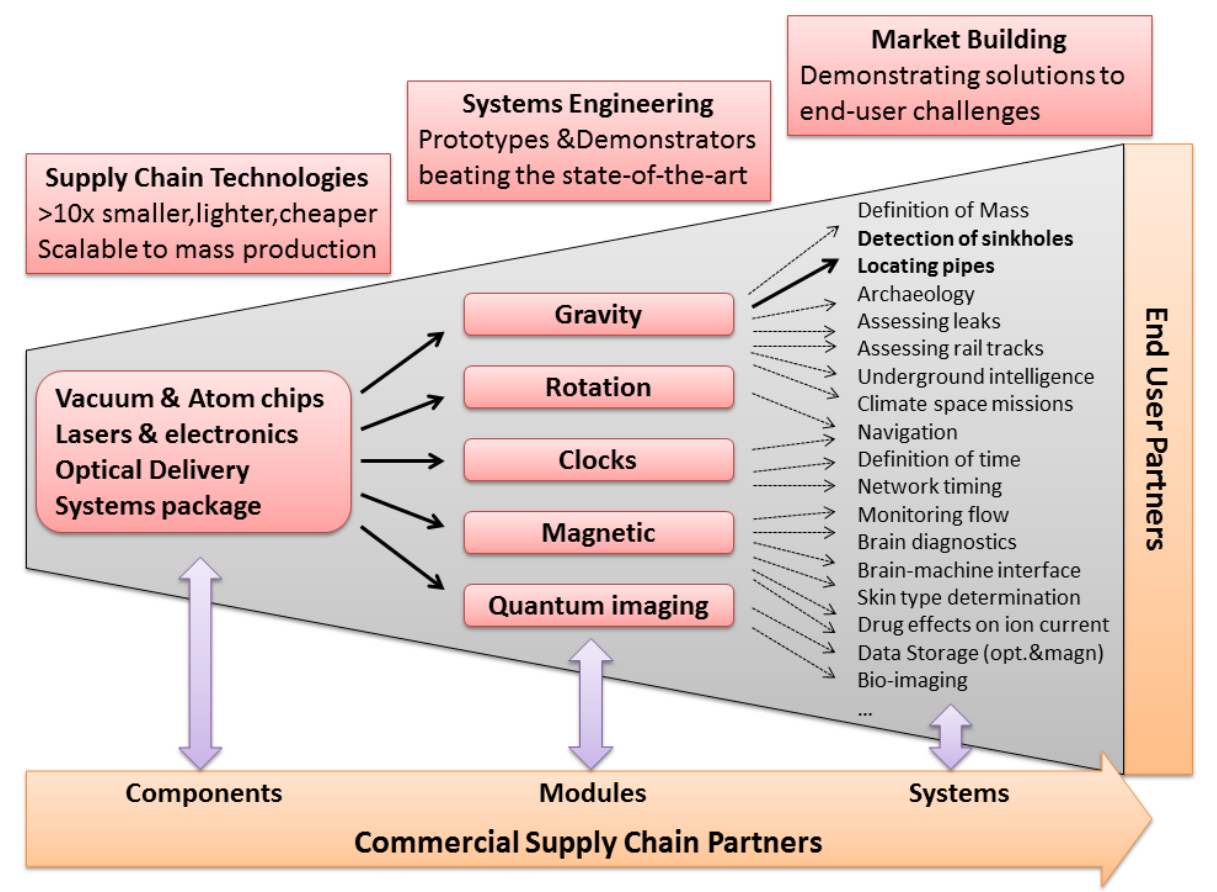

Figure 2. Key components of the UK National Quantum Technology Hub in Sensors and Metrology: (i) Supply Chain Technologies: A coordinated effort involving leading nanofabrication facilities and companies will move beyond the current state-of-the-art by developing mass-production scalable processes for self-contained vacuum, low-power atom chips, fully integrated laser and optical delivery systems, ASICS-ready electronics and additive manufacturing packaging technologies. Industry-involvement ensures that the novel technologies will be made commercially available. (ii) Systems Engineering: Under the supervision of industry, the Hub develops production-compatible technology demonstrators which surpass any classical instrument. A systems engineer facilitates standardization, defining interfaces and providing documentation for efficient knowledge transfer to commercialization partners. (iii) Market Building: The Hub aims to open new markets by running end-user driven, engineering-led activities demonstrating the disruptive potential of quantum sensors in various applications. The bold arrow indicates the activity of gravity in Civil Engineering acting as an example.

\section{SUPPLY CHAIN TECHNOLOGIES}

The Hub aims to facilitate the formation of a supply chain for the technologies developed within the Hub, opening them for commercialization by companies such that the entire cold atom and ion community will benefit and mass-production scale commercial quantum sensor manufacturers can be established.

The central aim of the technology research of our Hub is to facilitate quantum sensors which are much cheaper and smaller than the current state-of-the-art at comparable precision. This will allow crossing the barrier between the current limited markets e.g. in geophysics and large-platform navigation and open up completely new markets, moving towards the mass-market level and an enormous economic and social impact.

\subsection{Lasers}

In a joint effort by the nano-fabrication facilities in Glasgow (GGN), cold atom/ion experts across the Hub and laser companies, we aim to deliver the world's first wafer-scale integrated laser systems for atom cooling and interferometry including experiment control electronics. We anticipate integrated semiconductor laser systems at $<1 \%$ of the cost and $<0.1 \%$ of the volume as compared to conventional laser systems for atom interferometry and general laser cooling. The integrated laser system development relies on high yield achieved at GGN, their expertise in integrated systems at telecoms wavelength ${ }^{1}$, narrow linewidth DFB lasers ${ }^{2}$ as well as their experience of 700 to $900 \mathrm{~nm}_{\text {lasers }}^{3}$. Systems will be made commercially available via our industry partners. 
In addition the Hub will provide tailored lasers for quantum sensors with specifications outside typical semiconductor ranges. It will be based on VECSEL technology, which is currently replacing several high-power commercial systems due to its compactness, robustness, stability and spectral flexibility. Examples are compact $1 \mathrm{~W} 461 \mathrm{~nm}$ and narrow linewidth $689 \mathrm{~nm}$ lasers for $\mathrm{Sr}$ optical lattice clocks and lasers for ion trapping. In addition We aim at a miniature coherent femtosecond frequency comb system for optical clock time-extraction. This work is based on experts with pioneering contributions to $\mathrm{cW}$ VECSELS utilising a broad range of III-V semiconductors and solid-state laser engineering ${ }^{4-6}$ and to femtosecond VECSELS ${ }^{7,8}$ with microring femtosecond comb experts in Sussex ${ }^{9,10}$. The Fraunhofer CAP will facilitate the transfer of these systems to interested commercialization partners.

\subsection{Atomics package}

The Atomics Package creates the environment for the atoms/ions used as probe particles: vacuum, magnetic/electric field generation and optical interfaces. Our Hub brings together experts on atom/ion chip development, self-contained vacuum systems and silica photonics, to provide compact, integrated and robust systems that are scalable for mass-production. Based on our state-of-the-art ion chips with world-leading voltage breakdown ${ }^{11}$, we will deliver ion array chips based on our pioneering work ${ }^{12}$ in order to overcome the single ion limit in magnetometry and, potentially, clocks. We use more than 60 years of industrial vacuum electronics experience and detailed cold-atom vacuum developments ${ }^{13}$ to develop self-contained pump-less vacuum enclosures, which can be produced at a low cost and in large numbers. Where appropriate these will incorporate a novel laser-controlled and fully UHV compatible atom source invented in Birmingham (UK patent application No 1405258), atom chips and grating MOT designs. The hub combines expertise in semiconductor materials/quantum device development with advances in materials and atom-chip architectures/fabrication techniques ${ }^{14}$ to produce compact, low-noise, UHV compatible, atom chips for rotation sensing and magnetometry. In order to overcome the limitation of the current state-of-the-art optical fiber telescope systems to provide a smaller form factor, we will develop flat integrated waveguide to $\mathrm{cm}$-sized transverse output couplers. Utilizing recently develop tilted UV gratings writing techniques ${ }^{15}$ we will develop flat integrated waveguide to $\mathrm{cm}$-sized beam transverse output couplers, which can be applied flush to vacuum windows or potentially replace the window itself. The gratings will make use of exquisite control expertise to create anodized and phase controlled couplers for converting single mode waveguide inputs into carefully tailored beam profiles ${ }^{16}$.

\subsection{System package}

Combining the sensor components into a compact and robust system is a complex engineering task, which in the current state-of-the art requires a substantial manufacturing effort with significant compromises owing to machinability. We bring together the world leading advanced manufacturing expertise from the national EPSRC Centre for Innovative Manufacturing in Nottingham and the Additive Manufacturing and the Interdisciplinary Research Centre for Materials Processing in Birmingham Bham to overcome these limitations. We explore additive manufacturing techniques to deliver 3D printed atom chip base structures and topologically-optimised overall sensor packages including, where necessary, magnetic shields.

\section{PROTOTYPING AND SKILLS}

The hub aims to demonstrate the performance all supply chain technologies by the development of sensor prototypes surpassing any classical sensor. In this process it will train over 100 students and researchers from academia and industry in the skills needed to build, optimize and operate quantum sensors.

\subsection{Gravity Sensors}

In the area of absolute gravity sensors a low-volume, high-value market exists for geophysics and defence applications, which is targeted by first emerging spin-offs. The Hub aims to open new high-volume markets enabled by the reduction in size and cost promised by our technology platform. We will target two main areas: (i) absolute gravity sensors for metrology to contribute to the national definition of the $\mathrm{kg}$, in collaboration with our partner NPL, (ii) gravity gradient and gravity array sensors for underground mapping. The technology will be based on free-fall atom interferometry as the currently most precise available method with potential additions of cavity-enhanced large-momentum beamsplitters. Ultimately the Hub aims to integrate the supply chain technology outputs into next-generation $<101$ gravity sensor units with nano-g sensitivity and demonstrate array operation for gravitational imaging. 


\subsection{Magnetic Sensors}

Our hub aims at the most precise magnetic sensors from the microscale to macroscopic scales. Based on previous demonstration work we will develop a cold atom magnetic microscope ${ }^{17,18}$ with $1 \mathrm{D}$-imaging over mm-scale with micron resolution and stroboscopic analysis of dynamic processes and an ion array ${ }^{12}$ gradient magnetometer device for $\mathrm{mm}-\mathrm{cm}$ scale with noise suppression, which have no counterparts on the market. In addition the Hub works on $>\mathrm{cm}$ scale magnetic sensing with thermal atoms in microcells for operation at sensitivities competitive with superconducting quantum interference devices (SQUIDs) and significantly reduced cost and environmental demands.

\subsection{Rotation Sensors}

To our knowledge, there is currently no commercial cold atom sensor for rotation measurements, as precision laboratory demonstrations have so far been based on freely flying atoms in large and delicate setups. The Hub aims to overcome technological challenges in cold atom rotation sensors by developing new pulse techniques ${ }^{19}$ and use them to demonstrate a miniature cold atom gyroscope; in parallel, we shall introduce guiding schemes that allow the apparatus to be shrunk massively. As no clear optimum solution is available, we will start from our pioneering suggestions in magnetic $^{20,21}$ and diffractive optical ${ }^{22}$ atom guides and our developments driving guided rotation sensors in the EU project MatterWave: we will develop alternative schemes such as microwave based waveguides for rotation sensing ${ }^{23}$. The selection process of a final technology will be aided by developing an industry-compatible systems simulation based on our world-leading optical interferometry simulation programme Finesse (http://www.gwoptics.org/finesse/). The selected technology will be further developed into a compact device aiming at a sensitivity of $20 \times 10^{-10} \mathrm{rad} \mathrm{s}^{-1} \mathrm{~Hz}^{-1 / 2}$.

\subsection{Clocks}

Atomic clocks based on hot atoms have been commercially available for some time and are e.g. used in communication networks, in navigation applications as well as research labs. We will follow two main strands in developing a new generation of cold-atom based clocks: (i) A compact, cold atom microwave clock with sensitivity at $10^{-13}$ level based on our single-beam grating technology ${ }^{24}$ improving in cost, size and stability over the current state-of-the-art in commercial atomic clocks. (ii) An optical clock with a sensitivity of $10^{-16}$ in a robust portable version based on $\mathrm{Sr}$ in a magical optical lattice. These activities are complemented by the NPL development, under UK dstl funding, of both miniature thermal atom and cold ion microwave clocks, and of a compact ultra-stable clock laser for Sr atoms, which builds on NPL's Sr atom optical lattice clock $^{25}$

\subsection{Quantum Imaging}

Based on previous work by V. Boyer ${ }^{26}$ (patent US 7453626 B2); we will develop compact quantum sources of light showing multi-spatial mode squeezing and this enabling imaging applications at ultra-low noise levels.

\section{MARKET BUILDING}

The Hub engages with leading engineers and medical practitioners to demonstrate the applications and advantages of our prototypes in real-world applications in close collaboration with end-user partners. An example is the Civil Engineering area, where underground mapping is crucial to assess the state and location of sub-surface infrastructure. This provides an example of the type of demonstration activities we aim to deliver in the Hub with interested end-user industry partners. We aim to put a gravity gradient quantum sensor prototype into the hands of engineers to test in a real-world environment motivated by commercial problems, triggering markets and feeding back practical experience into the sensor development.

Initially, the quantum sensors emerging from our Hub will compete in an existing specialized market worth $\sim £ 20 \mathrm{M} /$ year. However, due to the $>10$-fold cost reduction that our supply chain technologies will yield, we expect disruptive uses to emerge (fostered by our demonstration activities) and the market for quantum sensors to grow to $\sim £ 1$ bn/year on a $15-20$ year time scale. An example is the construction industry market, where our industry co-creation event, held at the Royal Academy of Engineering in May 2014 and attended by 50 industrialists, revealed that an entry threshold of $\sim £ 30 \mathrm{k}$ per sensor would open a market of 10,000 gravity sensing units per year. Similarly, inertial navigation units for aircraft and ships would have to compete with current technology costing several $£ 10 \mathrm{k}$. At this price level, magnetic sensor systems for healthcare would be used in many hospitals and medical practices worldwide. Targeting sensor production at these 
markets will bring further price reductions until, at the $<£ 1 \mathrm{k}$ level, the multi-million unit consumer markets are reached including in car navigation, magnetic brain-machine/gaming interfaces, and hand-held accelerometers.

In addition to economic potential, sensors have enormous potential impact on society. Examples include: gravity sensors to locate underground pipes and so avoid unnecessary roadworks, monitor integrity under flooding, uncover history by imaging underground remnants, or monitor climatic change by satellite surveillance; magnetic sensors contributing to dementia research, determining skin types and potentially enabling the brain to communicate directly with computers.

\section{OUTLOOK AND ACKNOWLEDGEMENTS}

The UK National Quantum Technology Hub in Sensors and Metrology is now approaching the realization of the first prototypes, opening up exciting new opportunities in engineering research for real-world applications. We are looking forward to growing collaborations, exploring the benefits ultra-precise sensors for gravity, rotation, magnetic fields and time, as well as multimode-squeezed light sources could bring to future technologies. At the same time we aim to establish a knowledge transfer chain, moving exciting advanced quantum sensing schemes into practical use.

We gratefully acknowledge funding from the Engineering and Physical Sciences Research Council EPSRC under grant number EP/M013294/1 and from Dstl under contract numbers DSTLX-1000095040 and DSTLX-1000094497.

\section{REFERENCES}

[1] Zanola, M., Strain, M., Giuliani, G. and Sorel, M., "Monolithically integrated DFB lasers for tunable and narrow linewidth millimeter-wave generation", IEEE J. Select Topics Quantum Elec. 19, 1500406 (2013).

[2] Hou, L., Haji, M., Akbar, J., and Marsh, J. H., "Narrow linewidth laterally coupled $1.55 \mu \mathrm{m}$ AlGaInAs/InP distributed feedback lasers integrated with a curved tapered semiconductor optical amplifier", Opt. Letts. 37, 4525 (2012).

[3] Sorel, M., Giuliani, G., Scirè, A., Miglierina, R., Donati, S., Fellow and Laybourn, P. J. R., "Operating Regimes of GaAs-AlGaAs Semiconductor Ring Lasers: Experiment and Model”, IEEE J. Quant. Elec. 39, 1187 (2003).

[4] Calvez, S,. J.E. Hastie, J. E., Guina, M., Okhotnikov, O. G. and M.D. Dawson, M. D., "Semiconductor disk lasers for the generation of visible and ultraviolet radiation", Laser \& Photon. Rev. 3, 407 (2009).

[5] Parrotta, D. C., Kemp, A. J., Dawson, M. D. and Hastie, J. E., "Tunable continuous-wave diamond Raman laser", Opt. Exp. 19, 24165 (2011).

[6] Pabœuf, D., Schlosser, P. J. and Hastie, J. E. "Frequency stabilization of an ultraviolet semiconductor disk laser", Opt. Lett. 38, 1736 (2013).

[7] Quarterman, A. H., Wilcox, K. G., Apostolopoulos, V., Mihoubi, Z., Elsmere, S. P., Farrer, I., Ritchie, D. A., Tropper, A. "A passively mode-locked external-cavity semiconductor laser emitting 60 -fs pulses", Nat. Phot. 3 , 729 (2009).

[8] Wilcox, K. G., Tropper, A. C., Beere, H. E., Ritchie, D. A., Kunert, B., Heinen, B. and Stolz, W., “4.35 kW peak power femtosecond pulse mode-locked VECSEL for supercontinuum generation", Opt. Exp. 2121599 (2013).

[9] Peccianti, M., Pasquazi, A., Park, Y., Little, B. E., Chu, S. T., Moss, D. J., and Morandotti, R., ” Demonstration of a stable ultrafast laser based on a nonlinear microcavity", Nat. Commun, 3765 (2012).

[10] Peccianti et al. Patent US 20130156051 A1.

[11] Sterling, R. C., Hughes, M. D., Mellor, C. J. and Hensinger, W. K., "Increased surface flashover voltage in microfabricated devices", Appl. Phys. Lett. 103, 143504 (2013).

[12] Sterling, R. C., Rattanasonti, H., Weidt, S., Lake, K., Srinivasan, P., Webster, S. C., Kraft, M., and Hensinger, W. K., "Fabrication and operation of a two-dimensional ion-trap lattice on a high-voltage microchip", Nat. Comm. 5, 3637 (2014).

[13] J. Rushton, J., Aldous, M. and Himsworth, M., ArXiV: 1405.3148.

[14] Sinuco-León, G., Kaczmarek, B., Krüger, P. and Fromhold, T. M., " Atom chips with two-dimensional electron gases: Theory of near-surface trapping and ultracold-atom microscopy of quantum electronic systems", Phys. Rev. A 83, 021401 (2011).

[15] Xiong, C., Pernice, W., Ryu, K. K., Schuck, C., Fong, K. Y., Palacios, T. and Tang, H. X., "Integrated GaN photonic circuits on silicon (100) for second harmonic generation”, Optics Express 19, 10462 (2011). 
[16] Sima, C., Gates, J. C., Rogers, H. L., Mennea, P. L., Holmes, C., Zervas, M. N. and Smith P. G. R., "Ultra-wide detuning planar Bragg grating fabrication technique based on direct UV grating writing with electro-optic phase modulation", Optics Express 21, 15747 (2013).

[17] Wildermuth, S., Hofferberth, S., Lesanovsky, I., Haller, E., Andersson, L. M., Groth, S., Bar-Joseph, I., Krüger, P. and Schmiedmayer, J., "Bose-Einstein condensates: Microscopic magnetic-field imaging", Nature 435, 440 (2005).

[18] Wildermuth, S., Hofferberth, S., Lesanovsky, I., Groth, S., Krüger, P. and Schmiedmayer, J., "Sensing electric and magnetic fields with Bose-Einstein condensates", Appl. Phys. Lett. 88, 264103 (2006).

[19] Dunning, A., Gregory, R., Bateman, J., Cooper, N., Himsworth, M., Jones, J. A. and Freegarde, T., "Composite pulses for interferometry in a thermal cold atom cloud", Phys. Rev. A 90, 033608 (2014).

[20] Fernholz, T., Gerritsma, R., Krüger, P. and Spreeuw, R. J. C., "Dynamically controlled toroidal and ring-shaped magnetic traps", Phys. Rev. A 75, 063406 (2007).

[21] Griffin, P. F., Riis, E. and Arnold, A. S., "Smooth inductively coupled ring trap for atoms", Phys. Rev. A 77, 051402(R) (2008).

[22] Henderson, V. A., Griffin, P. F., Riis, E. and Arnold, A. S., "Comparative simulations of Fresnel holography methods for atomic waveguides", New J Phys 18, 025007 (2016).

[23] Sinuco-León, G. A., Burrows, K. A., Arnold, A. S. and Garraway, B. M., "Inductively guided circuits for ultracold dressed atoms", Nat. Commun. 5, 5289 (2014).

[24] Nshii, C. C., Vangeleyn, M., Cotter, J. P., Griffin, P. F., Hinds, E. A., Ironside, C. N., See, P., Sinclair, A. G., Riis, E. and Arnold A. S., "A surface-patterned chip as a strong source of ultracold atoms for quantum technologies", Nature Nanotechnology 8, 321 (2013).

[25]Hill, I. R., Hobson, R., Bowden, W., Bridge, E. M., Donnellan, S., Curtis, E. A. and Gill, P., "A low maintenance Sr optical lattice clock" Proc. $8^{\text {th }}$ Symp. Freq. Stds. \& Metrology, IOP Conf Series 2016; arXiv; 1602.05810 .

[26] Marino, A. M., Pooser, R. C., Boyer, V. and Lett, P. D., "Tunable delay of Einstein-Podolsky-Rosen entanglement", Nature 457, 859 (2009). 